\title{
Ground states of semilinear elliptic equations: a geometric approach
}

\author{
by
}

\author{
Rodrigo BAMÓN ${ }^{\mathrm{a}}$, Isabel FLORES ${ }^{\mathrm{a}}$, Manuel del PINO ${ }^{\mathrm{b}}$ \\ ${ }^{a}$ Departamento de Matemáticas, Facultad de Ciencias, Universidad de Chile, \\ Casilla 653 Correo 1, Santiago, Chile \\ b Departamento de Ingeniería Matemática and Centro de Modelamiento Matemático, \\ UMR2071 CNRS-UChile, Universidad de Chile, Casilla 170 Correo 3, Santiago, Chile
}

Manuscript received 2 July 1999, revised 2 February 2000

ABSTRACT. - We consider the problem

$$
\begin{gathered}
\Delta u+u^{p}+u^{q}=0, \quad \text { in } \mathbb{R}^{N} \\
0<u(x) \rightarrow 0 \quad \text { as }|x| \rightarrow+\infty,
\end{gathered}
$$

where $1<p<(N+2) /(N-2)<q$. We prove that if $q$ is fixed and we let $p$ approach $(N+2) /(N-2)$ from below, then this problem has a large number of radial solutions. A similar fact takes place if we fix $p>N /(N-2)$ and then let $q$ approach $(N+2) /(N-2)$. If we fix $q$ and then let $p$ be close enough to $N /(N-2)$ then no solutions exist. (C) 2000 L'Association Publications de l'Institut Henri Poincaré. Published by Elsevier B.V. All rights reserved

RÉSUMÉ. - On considère le problème de trouver des solutions de l'equation elliptique

$$
\Delta u+u^{p}+u^{q}=0, \quad \text { dans } \mathbb{R}^{N}
$$

avec

$$
0<u(x) \rightarrow 0 \quad \text { lorsque }|x| \rightarrow+\infty,
$$

où $1<p<(N+2) /(N-2)<q$. Si l'on fixe $q$ et $p$ augmente et tend vers $(N+2) /(N-2)$ alors il'y a un grand nombre des solutions radials. 
On peut obtenir un résultat analogue si l'on fixe $p>N /(N-2)$ et $q$ s'approche de $(N+2) /(N-2)$. En plus, si l'on fixe $q$ et l'on prend $p$ assez proche de $N /(N-2)$ alors il n'existe pas de solution.

(C) 2000 L'Association Publications de l'Institut Henri Poincaré. Published by Elsevier B.V. All rights reserved

\section{INTRODUCTION}

In this work we consider the problem of finding positive solutions of the following semilinear elliptic equation in $\mathbb{R}^{N}$.

$$
\begin{gathered}
\Delta u+u^{p}+u^{q}=0, \\
u(x) \rightarrow 0 \quad \text { as }|x| \rightarrow+\infty .
\end{gathered}
$$

Here $\Delta$ denotes the Laplacian operator in $\mathbb{R}^{N}, N \geqslant 3$. We also assume that the powers $p$ and $q$ are respectively sub and supercritical, namely

$$
1<p<\frac{N+2}{N-2}<q \text {. }
$$

A solution of (1.1)-(1.2) is usually called a ground state of (1.1). It is natural to search for radially symmetric ground states $u=u(|x|)$ of (1.1), so that $u(r)$ satisfies the ordinary differential equation

$$
\begin{gathered}
u^{\prime \prime}+\frac{N-1}{r} u^{\prime}+u_{+}^{p}+u_{+}^{q}=0, \quad r>0 \\
u^{\prime}(0)=0, \quad 0<u(r) \rightarrow 0 \quad \text { as } r \rightarrow+\infty .
\end{gathered}
$$

Here we have denoted $u_{+}=\max \{u, 0\}$. In the case of a nonlinearity constituted by a pure power, namely $p=q$ in (1.1), the role of the critical exponent in the problem of existence of positive ground states is well understood. If $p<(N+2) /(N-2)$, no positive solutions exist, see [5], while if $p=(N+2) /(N-2)$ all positive solutions are necessarily radial around some point, see [6]. At this exponent, as well as for $p>$ $(N+2) /(N-2)$, radial ground states are constituted by a one-parameter family of functions. More precisely, for every $\alpha>0$, the solution $u(r)$ of the initial value problem (1.4) with $p=q, u^{\prime}(0)=0, u(0)=\alpha>0$ is a ground state. 
We also notice that in case (1.3), it follows from a result in [4] that all solutions of (1.1)-(1.2) which decay at a sufficiently fast rate are necessarily radial around some point; this is however not known for all ground states.

A question raised by W.-M. Ni is the following: Are there radial ground states of (1.1)-(1.2) under the restriction (1.3)? Given the completely different pictures exhibited by purely subcritical and purely supercritical nonlinearities, an answer is not obvious. An interesting example was discovered by Lin and $\mathrm{Ni}$ in [10]. If $p$ and $q$ satisfy (1.3) and additionally $q=2 p-1$, then there is an explicit solution of the form $u(r)=A(B+$ $\left.r^{2}\right)^{-1 /(p-1)}$, where $A$ and $B$ are positive constants depending on $p$ and $N$. The question of existence of ground states in the general range (1.3) has remained however widely open.

In order to state our main results concerning this question, we need some definitions. A positive solution $u(r)$ of $(1.4)$ in $(0, \infty)$ is said to have slow decay if

$$
u(r)=A r^{-2 /(p-1)}+\mathrm{o}\left(r^{-2 /(p-1)}\right) \quad \text { as } r \rightarrow+\infty
$$

for some positive constant $A$. On the other hand, $u(r)$ is said to have fast decay if

$$
u(r)=\mathrm{O}\left(r^{-(N-2)}\right) \quad \text { as } r \rightarrow+\infty .
$$

Thus $u(r)$ is said to be a radial ground state of (1.1) if it is finite up to $r=0$ with $u^{\prime}(0)=0$. We call $u(r)$ a radial singular ground state if instead $u(r) \rightarrow+\infty$ as $r \rightarrow 0^{+}$. It can be shown that these are indeed all possible behaviors of a positive solution of (1.4). We remark that in this language, in the case of a pure power $p=q$, ground states have slow decay in the supercritical case, while they have fast decay at the critical exponent. Also, Lin and Ni's example is a ground state of slow decay.

THEOREM 1.1. - (a) Let $q>(N+2) /(N-2)$ be fixed. Then, given any integer $k \geqslant 1$, there exists a number $p_{k}<(N+2) /(N-2)$ such that if $p_{k}<p<(N+2) /(N-2)$, then (1.1) has at least $k$ radial ground states with fast decay.

(b) Let $N /(N-2)<p<(N+2) /(N-2)$ be fixed. Then, given any integer $k \geqslant 1$, there exists a number $q_{k}>(N+2) /(N-2)$ such that if $(N+2) /(N-2)<q<q_{k}$, then (1.1) has at least $k$ radial ground states with fast decay.

A nonexistence counterpart of the above result is the following. 
THEOREM 1.2. - Let $q>(N+2) /(N-2)$ be fixed. Then there is a number $\bar{p}>N /(N-2)$ such that if $1<p<\bar{p}$ then there are neither radial ground states nor radial singular ground states of (1.1).

We observe that this nonexistence result is optimal, in the sense that for $q=2 p-1$ there are ground states, and $(N+2) /(N-2)=$ $2 N /(N-2)-1$.

As for existence of singular ground states or slow-decay ground states, we have the following result.

THEOREM 1.3. - (a) Given $q>(N+2) /(N-2)$, there exists an increasing sequence of numbers $p_{1}<p_{2}<\cdots$ with $p_{k} \uparrow(N+2) /(N-2)$ such that if $p=p_{k}$ then there is a radial singular ground state of (1.1), with either slow or fast decay.

(b) Given $N /(N-2)<p<(N+2) /(N-2)$, there exists a decreasing sequence of numbers $q_{1}>q_{2}>\cdots$ with $q_{k} \downarrow(N+2) /(N-2)$ such that if $q=q_{k}$ then there is either a slow decay ground state or a slow decay singular solution.

The proof of Theorem 1.1 is based on the following key fact.

Proposition 1.4. - Assume that Eq. (1.4) has a solution $u_{0}(r)$ defined and positive on an interval $\left(0, R_{0}\right)$ and a solution $u_{\infty}(r)$ defined and positive on an interval $\left(R_{\infty}, \infty\right)$. Assume also that $R_{\infty}, R_{0}, u_{0} \not \equiv u_{\infty}$ and that $u_{0}-u_{\infty}$ has at least $2 k+1$ zeroes in $\left(R_{\infty}, R_{0}\right)$ for some $k \geqslant 1$. Then there exist at least $k-1$ radial ground states with fast decay of (1.1).

For instance the proof of part (a) of Theorem 1.1 is thus reduced to showing that for each number $k$ the assumptions of this result indeed hold if we fix $q$ supercritical and then let $p$ be close enough from below to the critical exponent. Similarly for part (b).

The proof of this proposition, as well as those of the other results stated above, is largely based on a rather delicate phase-space analysis of a three dimensional autonomous first order system equivalent to Eq. (1.4), obtained after the so-called Emden-Fowler transformations. Loosely speaking, a ground state with fast decay will correspond to a heteroclinic orbit connecting two equilibria of the system with respectively a twodimensional unstable manifold and a two-dimensional stable manifold. The assumptions of Proposition 1.1 amount to the presence of two trajectories lying respectively on each of these surfaces, which wind around each other at least $k$ times. After a topological analysis, we show that this winding inherits at least $k-1$ distinct trajectories lying 
simultaneously on the two surfaces, so that $k-1$ heteroclinic orbits are present.

It should be remarked that the presence of slow-decay solutions, like that of Lin and Ni's example, is harder to be detected (and expected to be non-generic) due to the fact that they correspond to a heteroclinic orbit lying simultaneously on the two-dimensional unstable manifold above mentioned and the stable manifold of a third equilibrium point which is only one-dimensional. In fact, if such a solution exists, it is automatically unique. A similar fact holds for a singular ground state. This "nongenericity" makes us suspect that typically no much more than the assertion of Theorem 1.3 can be said concerning existence of singular or slow decay ground states. In this sense, Lin and Ni's example of a slow ground state may well represent just a big coincidence, though we do not know if this is the case. Instead the presence of fast decay ground states is topologically "robust". It should be mentioned that the setting of the heteroclinic orbits here detected, seems in principle suitable for the application of Conley index theory, see [11], since the sum of the dimensions of the stable and unstable manifolds of the equilibria is larger than the dimension of the space. However the multiplicity assertion seems hard to be obtained without the special topological analysis carried out here, not to mention the presence of a trivial heteroclinic representing the solution $u \equiv 0$.

Proposition 1.1 is a somewhat surprising type of "topological shooting", which seems applicable to a much broader class of nonlinearities, but we will not ellaborate about this point here.

To be noticed is the fact that fast-decay ground states have finite energy, so that one may expect them to be in principle workable via variational methods. On the other hand, this seems hard, at least through usual minmax characterizations. A point to be made is that a geometric approach like that devised in this paper may reveal in fairly natural ways a lot of the underlying structure of the problem. In this line we may also mention for instance the works [8,9] and [1], where dynamical systems tools have been used in the study of related equations.

The rest of this paper is organized as follows: In Section 2 we introduce the Emden-Fowler transformations and the equivalent first order system. We analyze its equilibria and describe locally the phase space near these points. In Section 3 we prove Proposition 1.1 via topological arguments, while in Section 4 we establish as a corollary the validity of Theorem 1.1. In Section 5 we prove the nonexistence result Theorem 1.2, and finally we prove Theorem 1.3 in Section 7. 


\section{THE FIRST ORDER SYSTEM}

We consider the classical Emden-Fowler transformation

$$
x(t)=\left.r^{2 /(q-1)} u(r)\right|_{r=\mathrm{e}^{t}}
$$

which transforms Eq. (1.4) into the equivalent problem

$$
x^{\prime \prime}+\alpha x^{\prime}+x_{+}^{q}+\mathrm{e}^{\gamma t} x_{+}^{p}-\beta x=0, \quad-\infty<t<+\infty,
$$

where

$$
\alpha=N-2-\frac{4}{q-1}, \quad \beta=\frac{2}{q-1}\left(N-2-\frac{2}{q-1}\right), \quad \gamma=2 \frac{q-p}{q-1} .
$$

Standard calculations show that finding a positive radial ground state of (1.1), namely a solution of (1.4)-(1.5) is equivalent to finding a positive solution $x(t)$ in $\mathbb{R}$ of (2.2) such that

$$
x(t) \rightarrow 0 \quad \text { as } t \rightarrow \pm \infty .
$$

Introducing the variables $y=x^{\prime}$ and $z=\mathrm{e}^{\gamma t}$, Eq. (2.2) becomes equivalent to the autonomous first order system

$$
\left\{\begin{array}{l}
x^{\prime}=y, \quad y^{\prime}=-\alpha y+\beta x-x_{+}^{q}-z x_{+}^{p}, \\
z^{\prime}=\gamma z, \\
z \geq 0 .
\end{array}\right.
$$

Our task is therefore equivalent to finding a solution $\mathbf{x}(t)=(x(t), y(t)$, $z(t))$ of this system, with $z(t)>0$, such that $\mathbf{x}(t) \rightarrow 0$ as $t \rightarrow-\infty$, while $(x(t), y(t)) \rightarrow(0,0)$ as $t \rightarrow+\infty$.

We observe that the plane $z=0$ is invariant under the flow associated to system (2.3). This plane contains the two singularities of the flow $O_{0}=(0,0,0)$ and $P_{0}=\left(\beta^{1 /(q-1)}, 0,0\right)$. For the flow restricted to this plane, $O_{0}$ is a hyperbolic saddle. $P_{0}$ is a hyperbolic attractor. They are connected by a heteroclinic orbit, precisely a branch of the unstable manifold of $O_{0}$ restricted to $z=0$. This orbit is transversal to $x=0$, see Fig. 1. This phase plane analysis (corresponding to the case of a single power) is actually well known. See for instance the appendix in [9] and its references. 


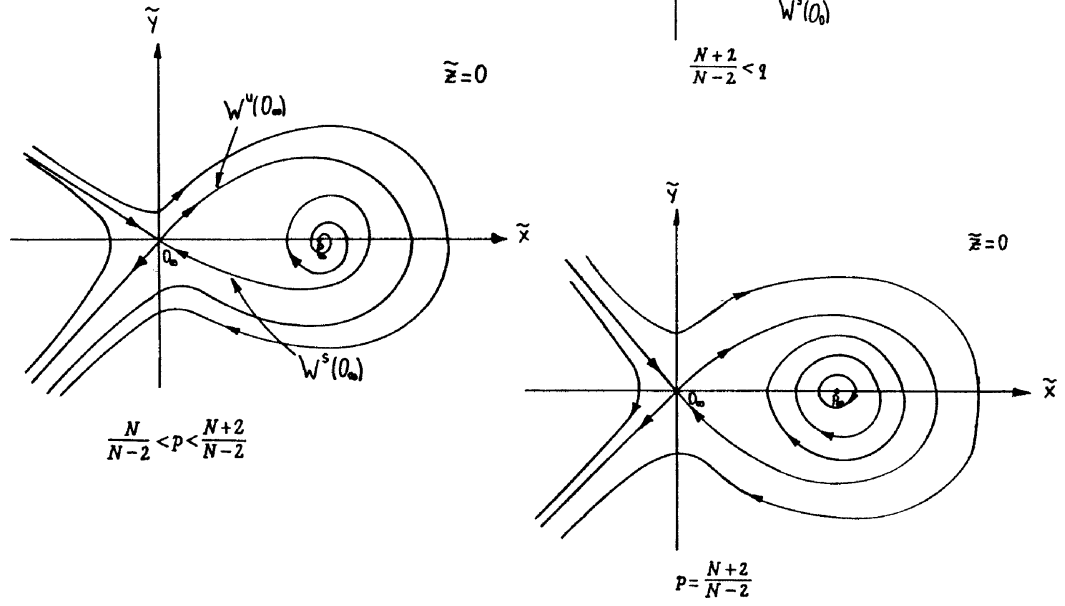

Fig. 1.

Let us now consider the behavior of the entire flow on the half-space $z \geqslant 0$ near these singularities. Linearizing the flow around $O_{0}$ one obtains one stable eigenvalue $2 /(q-1)-(N-2)<0$ with associated eigenvector $(1,2 /(q-1)-(N-2), 0)$ and two unstable eigenvalues $2 /(q-1)$ and $\gamma=2(q-p) /(q-1)$ with associated eigenvectors $(1,2 /(q-1), 0)$ and $(0,0,1)$. Thus, from standard invariant manifold theory, see for instance [7], $O_{0}$ has a two dimensional unstable manifold $W^{u}\left(O_{0}\right)$, constituted by all trajectories approaching $O_{0}$ as $t \rightarrow-\infty$, whose tangent plane is spanned by the two unstable eigenvectors. Moreover, it coincides with this plane for $x<0$. $W^{u}\left(O_{0}\right)$ contains the entire $z$-axis as well as the heteroclinic orbit on $z=0$ connecting $O_{0}$ and $P_{0}$. It is also transversal to the planes $z=0$ and $x=0$. Now, linearizing around the singularity $P_{0}$ we obtain the unstable eigenvalue $\gamma$ with associated eigenvector

$$
\left(-1,-\gamma, \frac{\beta(q-1)+\alpha \gamma+\gamma^{2}}{\beta^{p /(q-1)}}\right) .
$$



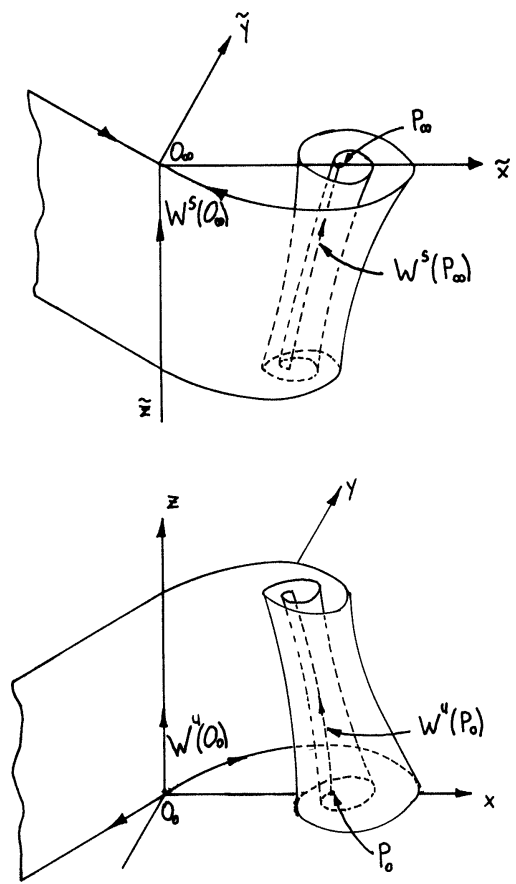

Fig. 2.

We also have the two stable eigenvalues

$$
\frac{-\alpha \pm \sqrt{\alpha^{2}-4 \beta(q-1)}}{2}
$$

with eigenfunctions contained in the plane $z=0$. The unstable manifold of $P_{0}$ for $z>0, W^{u}\left(P_{0}\right)$, is thus one-dimensional, and constituted by a single orbit, see Fig. 2.

In order to analyze the behavior of trajectories near $z=+\infty$ it is convenient to introduce also the additional transformation,

$$
\begin{aligned}
& \tilde{x}=x z^{1 /(p-1)}, \\
& \tilde{y}=\left(y+\frac{\gamma x}{p-1}\right) z^{1 /(p-1)}, \\
& \tilde{z}=\frac{1}{z^{(q-1) /(p-1)}} .
\end{aligned}
$$


which makes the system equivalent to

$$
\left\{\begin{array}{l}
\tilde{x}^{\prime}=\tilde{y}, \\
\tilde{y}^{\prime}=\tilde{\alpha} \tilde{y}+\tilde{\beta} \tilde{x}-\tilde{x}_{+}^{p}-\tilde{z} \tilde{x}_{+}^{q}, \\
\tilde{z}^{\prime}=-\tilde{\gamma} \tilde{z}
\end{array}\right.
$$

with

$$
\begin{gathered}
\tilde{\alpha}=\frac{4}{p-1}-(N-2), \quad \tilde{\beta}=\frac{2}{p-1}\left(N-2-\frac{2}{p-1}\right), \\
\tilde{\gamma}=2 \frac{q-p}{p-1} .
\end{gathered}
$$

This transformation corresponds to using the exponent $p$ instead of $q$ in the Emden-Fowler transformation (2.1), which is expected to reflect better the behavior of a ground state at infinity. In fact, the effect of this transformation is to "blow-up" the "singularity" $(0,0, \infty)$ into the plane $\tilde{z}=0$. These new coordinates extend up to $\tilde{z}=0$, which is invariant under the flow associated to system (2.5). The singularities of this new flow are the points $O_{\infty}=(0,0,0)$ and $P_{\infty}=\left(\tilde{\beta}^{1 /(p-1)}, 0,0\right)$. For the flow restricted to this plane, $O_{\infty}$ is a hyperbolic saddle and $P_{\infty}$ is a hyperbolic repulsor. They are connected by a heteroclinic orbit which corresponds to a branch of the stable manifold of $O_{\infty}$ restricted to $\tilde{z}=0$, see Fig. 1 . As for the entire flow on $\tilde{z} \geqslant 0$ near these singularities, we obtain that $O_{\infty}$ has associated two stable eigenvalues $2 /(p-1)-(N-2)<0$ and $-\tilde{\gamma}=-2(q-p) /(p-1)$ with corresponding eigenvectors.

$(1,2 /(p-1)-(N-2), 0)$ and $(0,0,1)$, as well as one unstable eigenvalue $2 /(p-1)$ with eigenvector $(1,2 /(p-1), 0)$. Thus, $O_{\infty}$ has a two-dimensional stable manifold $\widetilde{W^{s}}\left(O_{\infty}\right)$ with tangent plane spanned by the two stable eigenvectors and it coincides with this plane for $\tilde{x}<0$. $\widetilde{W}^{s}\left(O_{\infty}\right)$ contains the entire $\tilde{z}$-axis as well as the heteroclinic orbit connecting $O_{\infty}$ and $P_{\infty}$. It is transversal to both planes $\tilde{z}=0$ and $\tilde{x}=0$. Similarly, $P_{\infty}$ has the stable eigenvalue $-\tilde{\gamma}$ with associated eigenvector

$$
\left(-1, \tilde{\gamma}, \frac{\tilde{\beta}(p-1)+\tilde{\alpha} \tilde{\gamma}+\tilde{\gamma}^{2}}{\tilde{\beta}^{p / q-1}}\right) .
$$


$P_{\infty}$ has also the two unstable eigenvalues

$$
\frac{\tilde{\alpha} \pm \sqrt{\tilde{\alpha}^{2}-4 \tilde{\beta}(p-1)}}{2}
$$

with eigenfunctions contained in the plane $\tilde{z}=0$. The stable manifold of $P_{\infty}, \widetilde{W}^{s}\left(P_{\infty}\right)$, is thus one dimensional, and constituted (for $\tilde{z}>0$ ) by a single orbit. The following result describes the asymptotic behavior of a trajectory of system (2.3) whose $x$-coordinate remains positive as $t \rightarrow-\infty$, and that of a trajectory of (2.5) whose $\tilde{x}$-coordinate remains positive as $t \rightarrow+\infty$.

LEMMA 2.1. - Let $\mathbf{x}(t)=\left(x(t), y(t), \mathrm{e}^{\gamma t}\right)$ be a solution of system (2.3). $\tilde{\mathbf{x}}(t)=\left(\tilde{x}(t), \tilde{y}(t), \mathrm{e}^{-\tilde{\gamma} t}\right)$ a solution of (2.5).

(a) Assume that $x(t)>0$ for all $-\infty<t<t_{0}$. Then the orbit of $\mathbf{x}$ is either contained in $W^{u}\left(O_{0}\right)$ or it coincides with $W^{u}\left(P_{0}\right)$.

(b) Assume that $\tilde{x}(t)>0$ for all $t_{0}<t<\infty$. Then the orbit of $\tilde{\mathbf{x}}$ is either contained in $\widetilde{W}^{s}\left(O_{\infty}\right)$ or it coincides with $\widetilde{W}^{s}\left(P_{\infty}\right)$.

Proof. $-x(t)$ satisfies the differential equation,

$$
x^{\prime \prime}+\alpha x^{\prime}+x_{+}^{q}+\mathrm{e}^{\gamma t} x_{+}^{p}-\beta x=0, \quad-\infty<t<+\infty,
$$

so that $u(r)=r^{-2 /(q-1)} x(\log r)$ satisfies

$$
-\left(r^{N-1} u^{\prime}(r)\right)^{\prime}=r^{N-1}\left(u^{p}(r)+u^{q}(r)\right) .
$$

Assume $u(r)$ is unbounded as $r \rightarrow 0$. Then there exists a decreasing sequence $\delta_{n} \rightarrow 0$ with $u^{\prime}\left(\delta_{n}\right) \leqslant 0$. From the above equation we get then that $u^{\prime}(r) \leqslant 0$ for all $r>0$. Also, integrating from $\delta_{n}$ to $r$ we get

$$
r^{N-1} u^{\prime}(r)=\delta_{n}^{N-1} u^{\prime}\left(\delta_{n}\right)+-\int_{\delta_{n}}^{r} s^{N-1}\left(u^{p}(s)+u^{q}(s)\right) \mathrm{d} s,
$$

so that

$$
r^{N-1} u^{\prime}(r) \leqslant-\left(r^{N}-\delta_{n}^{N}\right) C u^{q}(r)
$$

for all small $r>0$. Hence

$$
u(r) \leqslant C r^{-2 /(q-1)} .
$$


Assume first that there is a number $\delta>0$ with $x(t) \geqslant \delta$ as $t \rightarrow-\infty$. Stand now at any sequence $t_{n} \rightarrow-\infty$ then $x_{n}(t)=x\left(t+t_{n}\right)$ is uniformly bounded above, and below away from zero and satisfies

$$
x_{n}^{\prime \prime}+\alpha x_{n}^{\prime}+x_{n+}^{q}+\mathrm{e}^{\gamma\left(t+t_{n}\right)} x_{n+}^{p}-\beta x_{n}=0 .
$$

A standard compactness argument yields that $x_{n}$ has a subsequence, uniformly convergent over compacts to a solution positive and bounded above and below away from zero of

$$
\bar{x}^{\prime \prime}+\alpha \bar{x}^{\prime}+\bar{x}^{q}-\beta \bar{x}=0, \quad-\infty<t<\infty .
$$

But, phase plane analysis shows that the only solution of this equation with that property is the constant $\bar{x} \equiv \beta^{1 /(q-1)}$. Since the sequence $t_{n}$ was arbitrary, it follows that $\mathbf{x}(t) \rightarrow P_{0}$ as $t \rightarrow-\infty$, in other words this trajectory lies in $W_{u}\left(P_{0}\right)$. Assume now that there is a sequence $t_{n} \rightarrow-\infty$ such that $x\left(t_{n}\right) \rightarrow 0$ but that $x(t) \nrightarrow 0$ as $t \rightarrow-\infty$. Then we may find a second sequence $t_{n}^{\prime} \rightarrow-\infty$ such that $0<\delta<x\left(t_{n}^{\prime}\right)<$ $\beta^{1 /(q-1)} / 2$ and $x^{\prime}\left(t_{n}^{\prime}\right) \leqslant 0$. Then, analogously to the previous case, we have that a subsequence of $x\left(t+t_{n}^{\prime}\right)$ which converges uniformly over compacts to a bounded, positive solution $\bar{x}$ of (2.6) such that $\bar{x}^{\prime}(0) \leqslant 0$, $\bar{x}(0)<\beta^{1 /(q-1)} / 2$. However, phase plane analysis again yields that no such solution exists. Hence $x(t) \rightarrow 0$ as $t \rightarrow-\infty$ which in turn yields also $x^{\prime}(t) \rightarrow 0$ using the second order equation, so that $\mathbf{x}(t) \rightarrow O_{0}$ as $t \rightarrow-\infty$. We conclude that this trajectory then lies in $W^{u}\left(O_{0}\right)$, and the proof of the first assertion of the lemma is complete.

The proof of the second assertion is very similar. We claim that there is a number $a>0$ with $u^{\prime}(r) \leqslant 0$ for all $r>a$. Indeed, assume the oppoite, namely that there is a sequence $r_{n} \rightarrow+\infty$ with $u^{\prime}\left(r_{n}\right)>0$. Then

$$
r_{n}^{N-1} u^{\prime}\left(r_{n}\right)-r^{N-1} u^{\prime}(r)=-\int_{r}^{r_{n}}\left(u^{p}+u^{q}\right) s^{N-1} \mathrm{~d} s .
$$

Hence

$$
\int_{r}^{\infty}\left(u^{p}+u^{q}\right) s^{N-1} \mathrm{~d} s \leqslant r^{N-1} u^{\prime}(r)
$$

which inplies that, on the one hand, $u$ is nondecreasing, on the other that the left integral is finite. But this implies that $u \equiv 0$, a contradiction. 
Assume then that for $r>a u$ is nonincreasing. Then

$$
-u^{\prime}(r) \geqslant \frac{1}{r^{N-1}} \int_{a}^{r} u^{p}(s) s^{N-1} \mathrm{~d} s .
$$

It follows that

$$
-u^{\prime}(r) \geqslant \frac{r}{N} u^{p}(r)
$$

From here it easily follows that $u(r) \leqslant C r^{-2 /(p-1)}$ which implies that $\tilde{x}$ remains bounded as $t \rightarrow+\infty$. After this, the proof of the second assertion of the lemma follows in exact analogy as that of the first, now using the fact that $\tilde{x}$ satisfies the equation

$$
\tilde{x}^{\prime \prime}+\tilde{\alpha} \tilde{x}^{\prime}+\tilde{x}_{+}^{p}+\mathrm{e}^{-\tilde{\gamma} t} \tilde{x}_{+}^{q}-\tilde{\beta} x=0, \quad-\infty<t<+\infty .
$$

This finishes the proof.

The following intuitively clear fact will be important for further analysis.

LEMMA 2.2. - The unstable manifold of $P_{0}, W^{u}\left(P_{0}\right)$ is contained in the closure of the unstable manifold of $O_{0}, W^{u}\left(P_{0}\right)$. Similarly, the stable manifold of $P_{\infty}, \widetilde{W}^{s}\left(P_{\infty}\right)$ is contained in the closure of the stable manifold of $O_{\infty}, \widetilde{W}^{s}\left(P_{\infty}\right)$.

For the proof of this result, we refer to the remark after the proof of Lemma 5.1

Let us define the manifolds $W^{s}\left(O_{\infty}\right)$ and $W^{s}\left(P_{\infty}\right)$ as the intersection of $\widetilde{W}^{s}\left(O_{\infty}\right)$ and $\widetilde{W}^{s}\left(P_{\infty}\right)$ with $\tilde{z}>0$, expressed in terms of the original coordinates $x, y, z$, namely

$$
\begin{aligned}
& W^{s}\left(O_{\infty}\right)=\left\{(x, y, z) \mid(\tilde{x}, \tilde{y}, \tilde{z}) \text { given by }(2.4) \in \widetilde{W}^{s}\left(O_{\infty}\right), z>0\right\}, \\
& W^{s}\left(P_{\infty}\right)=\left\{(x, y, z) \mid(\tilde{x}, \tilde{y}, \tilde{z}) \text { given by }(2.4) \in \widetilde{W}^{s}\left(P_{\infty}\right), z>0\right\} .
\end{aligned}
$$

Let us observe then that a (nontrivial) trajectory $(x(t), y(t), z(t))$ which lies in $W^{u}\left(O_{0}\right)$ and simultaneously in $W_{s}\left(O_{\infty}\right)$ corresponds to a radial ground state of (1.1), in the sense that

$$
u(r)=r^{-2 /(q-1)} x(\log r)
$$


solves (1.4)-(1.5). In fact this is a fast decay ground state. If this trajectory lies in $W^{u}\left(O_{0}\right) \cap W^{u}\left(P_{\infty}\right)$, then this is a slow-decay ground state, in the sense of (1.6). These decay rates are easy to confirm via linearization around $O_{\infty}$ and $P_{\infty}$. In fact, the reciprocal is also true. Any ground state needs to be of one of the types just described in terms of the $x$ transformation.

We should remark that a ground state needs to remain positive if not identically zero. A trajectory which intersects the $x=0$ plane from the $x>0$ side, never crosses it back (observe that on $x<0$ the system is just linear.)

Finally, we observe that a singular ground state corresponds precisely to a trajectory lying in $W^{u}\left(P_{0}\right) \cap W^{u}\left(O_{\infty}\right)$ or $W^{u}\left(P_{0}\right) \cap W^{u}\left(P_{\infty}\right)$. We observe that there is at most one of such trajectories, since $W^{u}\left(P_{0}\right)$ is one-dimensional, and the system is not invariant in $z$-translations.

\section{THE PROOF OF PROPOSITION 1.1}

We shall kepp here the notation introduced in the previous section. What we have to show is that if the assumption of Proposition 1.1 is accomplished by certain number $k$, then there exist at least $k-1$ distinct trajectories in $W^{u}\left(P_{0}\right) \cap W^{s}\left(O_{\infty}\right)$.

Let $u_{0}(r)$ and $u_{\infty}(r)$ be solutions of (1.4) as in the statement of the proposition. We define $x_{i}(t)=\left.r^{-2 /(q-1)} u_{i}(r)\right|_{r=\mathrm{e}^{t}}, i=0, \infty$ and likewise $y_{i}(t)=x_{i}^{\prime}(t), z(t)=\mathrm{e}^{\gamma t}$, according to the transformation (2.4). Let us set

$$
\mathbf{x}_{i}(t)=\left(x_{i}(t), y_{i}(t), z(t)\right)
$$

We will also denote $t_{1}=\ln R_{\infty}, t_{2}=\ln R_{0}$. Since, by assumption, $x_{0}(t)>$ 0 for all $-\infty<t<t_{2}$, it follows from Lemma 2.1 that the orbit of $\mathbf{x}_{0}$ lies in $W^{u}\left(O_{0}\right)$ or it coincides with a branch of $W^{u}\left(P_{0}\right)$ (the latter is the case of $u_{0}$ a singular solution). Similarly, $\mathbf{x}_{\infty}$ is a trajectory lying either in $W^{s}\left(O_{\infty}\right)$ or in $W^{s}\left(P_{\infty}\right)$. The assumption of the proposition is that $x_{0}-x_{\infty}$ has at least $2 k+1$ simple zeros in the interval $\left(t_{1}, t_{2}\right)$, which amounts to the fact that the trajectories $\mathbf{x}_{0}(t)$ and $\mathbf{x}_{\infty}(t)$ wind around each other at least $k$ times. We will establish this below, with a precise definition. Since, as we saw in the previous section, $W^{u}\left(P_{0}\right)$ lies in the closure of $W^{u}\left(O_{0}\right)$, and $W^{s}\left(P_{\infty}\right)$ lies in the closure of $W^{s}\left(O_{\infty}\right)$, it follows that without loss of generality we may assume that $\mathbf{x}_{0}$ lies in $W^{u}\left(O_{0}\right)$, and $\mathbf{x}_{\infty}$ lies in $W^{s}\left(O_{\infty}\right)$. 
Moreover, if we assume that only a finite number of trajectories lie simultaneously in $W^{u}\left(O_{0}\right)$ and in $W^{s}\left(O_{\infty}\right)$ (otherwise an infinite number of ground states with fast decay automatically exist), then slightly perturbing $\mathbf{x}_{i}(t)$ to neighboring trajectories in $W^{u}\left(O_{0}\right)$, respectively in $W^{s}\left(O_{\infty}\right)$, we may also assume without loss of generality that these trajectories do not lie simultaneusly in the two manifolds.

We observe that the $z$-axis separates the manifold $W^{u}\left(O_{0}\right)$ into two components invariant under the flow, one of them a half-plane contained in $x<0$, the other a surface $W_{+}^{u}\left(O_{0}\right)$, which we define so that it contains the $z$-axis. Observe that $W_{+}^{u}\left(O_{0}\right)$ is not necessarily contained in $x \geqslant 0$. Let us observe that the trajectory $\mathbf{x}_{0}$ splits $W_{+}^{u}\left(O_{0}\right)$ into two components. Let us call $H_{0}$ the closure of the component which contains the $z$-axis.

Let us denote $U\left(z_{0}\right)=H_{0} \cap\left\{z=z_{0}\right\}$. Then $U\left(z_{0}\right)$ is a $C^{1}$ curve without self-intersections, whose endpoints are $\left(0,0, z_{0}\right)$ and the point of the trajectory $\mathbf{x}_{0}$ in the plane $\left\{z=z_{0}\right\}$.

Similarly, we see that the $z$-axis separates the manifold $W^{s}\left(O_{\infty}\right)$ into two components invariant under the flow, one of them a half-plane contained in $x<0$, the other a surface $W_{+}^{s}\left(O_{\infty}\right)$, which we define so that it contains the $z$-axis. Now, the trajectory $\mathbf{x}_{\infty}$ splits $W_{+}^{s}\left(O_{\infty}\right)$ into two components. Let us call $H_{\infty}$ the closure of the component which contains the $z$-axis. We denote $S\left(z_{0}\right)=H_{\infty} \cap\left\{z=z_{0}\right\}$.

Our goal is to prove that for certain $z_{0}$ the curves $U\left(z_{0}\right)$ and $S\left(z_{0}\right)$ intersect at least at $k-1$ points. Observe that these intersections will correspond to $k-1$ distinct trajectories lying simultaneously in $W^{s}\left(O_{\infty}\right)$ and $W^{u}\left(O_{0}\right)$, hence to $k-1$ radial ground states with fast decay of (1.1).

In order to do this, we need some preliminaries. We can lift a planar curve $\sigma(s), s \in[0,1]$, in $\mathbb{R}^{2} \backslash\left\{\left(x_{0}, y_{0}\right)\right\}$, to a curve $\bar{\sigma}(s)=(\theta(s), \rho(s))$ in the polar coordinates plane via the relation

$$
\sigma(s)=\left(x_{0}+\rho(s) \sin \theta(s), y_{0}+\rho(s) \cos \theta(s)\right) .
$$

We define the winding number of $\sigma$ around $\left(x_{0}, y_{0}\right)$ as the number

$$
W\left(\sigma,\left(x_{0}, y_{0}\right)\right)=\left[\frac{1}{2 \pi}(\theta(1)-\theta(0))\right],
$$

where [·] denotes integral part.

Next we consider two disjoint curves $\gamma_{1}$ and $\gamma_{2}$ in the 3-dimensional space which can be parametrized by the $z$-coordinate in the form

$$
\gamma_{i}(z)=\left(x_{i}(z), y_{i}(z), z\right), \quad z \in\left[z_{1}, z_{2}\right] .
$$




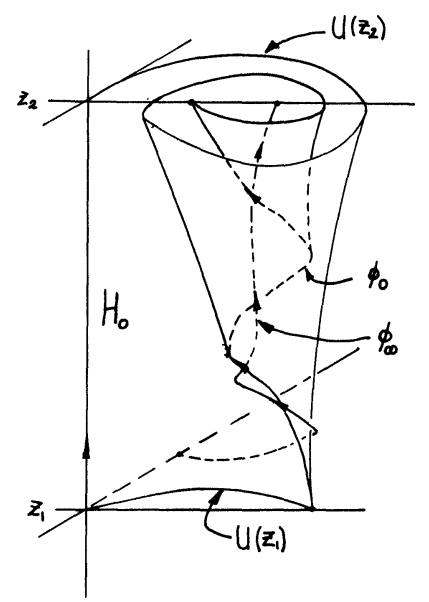

Fig. 3.

We define the linking number of $\gamma_{1}, \gamma_{2}$ in $\left[z_{1}, z_{2}\right]$ to be the integer $W(\sigma,(0,0))$, where $\sigma(z)=\left(x_{1}(z)-x_{2}(z), y_{1}(z)-y_{2}(z)\right), z \in\left[z_{1}, z_{2}\right]$. This number is obviously invariant under homotopies which preserve endpoints of the curves, keep the curves disjoint and preserve their $z$ coordinates.

Let $\phi_{i}(z)$ be a parametrization of the trajectory $\mathbf{x}_{i}, i=0, \infty$, via the $z$ coordinate, namely $\phi_{i}(z)=\mathbf{x}_{i}\left(\gamma^{-1} \log z\right)$. Fix numbers $z_{1}$ and $z_{2}$ and let $\sigma_{1}(s)$ and $\sigma_{2}(s), s \in[0,1]$, be one-to-one parametrizations respectively of $U\left(z_{1}\right)$ and $U\left(z_{2}\right)$, such that $\sigma_{1}(0)=\phi_{0}\left(z_{1}\right), \sigma_{1}(1)=\left(0,0, z_{1}\right)$ and $\sigma_{2}(0)=\left(0,0, z_{2}\right)$ and $\sigma_{2}(1)=\phi_{0}\left(z_{2}\right)$.

See Fig. 3 for a description of the linking situation we are concerned with.

The result of Proposition 1.1 is a direct consequence of the following three lemmas.

LEMMA 3.1. - There exists a number $\bar{z}_{1}>0$ such that for any $0<$ $z_{1}<\bar{z}_{1}$ and all $z_{2}>z_{1}$ the winding number of curve $\sigma_{2}$, contained in the plane $z=z_{2}$, around the point $\phi_{\infty}\left(z_{2}\right), W\left(\sigma_{2}, \phi_{\infty}\left(z_{2}\right)\right)$ equals $m-1$ or $m$, where $m$ is the linking number of the curves $\phi_{0}$ and $\phi_{\infty}$ in $\left[z_{1}, z_{2}\right]$.

LEMMA 3.2. - If $k$ is the number given by the assumption of Proposition 1.1, and $0<z_{1}<z_{2}$, then the linking number of the curves $\phi_{0}$ and $\phi_{\infty}$ in $\left[z_{1}, z_{2}\right]$, is at least $k$.

LEMMA 3.3. - If $z_{2}$ is chosen sufficiently large, then the curves $U\left(z_{2}\right)$ and $S\left(z_{2}\right)$ intersect at least $W\left(\sigma_{2}, P_{z_{2}}\right)$ times. 
We will devote the rest of this section to the proof of these results.

Proof of Lemma 3.1. - Let us observe that there is a unique value of $z$ for which $\phi_{\infty}(z)$ crosses the plane $x=0$. We choose $z_{1}$ to be this value, so that $\phi_{\infty}(z) \in\{x>0\}$ for all $z>z_{1}$.

Let $\varepsilon$ be a small fixed positive number. Let us define $\tilde{\phi}_{0}(z)$ to be the following curve

$$
\tilde{\phi}_{0}(z)= \begin{cases}\sigma_{1}\left(\frac{z-z_{1}}{\varepsilon}\right)+\left(0,0, z-z_{1}\right) & \text { if } z_{1}-\leqslant z<z_{1}+\varepsilon, \\ (0,0, z) & \text { if } z_{1}+\varepsilon \leqslant z \leqslant z_{2}-\varepsilon, \\ \sigma_{2}\left(\frac{z-\left(z_{2}-\varepsilon\right)}{\varepsilon}\right)+\left(0,0, z-z_{2}\right) & \text { if } z_{2}-\varepsilon<z \leqslant z_{2} .\end{cases}
$$

Next we check that if $\varepsilon$ is chosen small enough then $\phi_{0}$ and $\tilde{\phi}_{0}$ are homotopic inside the region

$$
\mathcal{S}=\left\{z_{1} \leqslant z \leqslant z_{2}\right\} \backslash \phi_{\infty}\left(\left[z_{1}, z_{2}\right]\right),
$$

leaving endpoints fixed and the $z$-coordinate invariant. In fact, let us consider the surface $H_{0}$ defined earlier in this section, constituted by the component of $W_{+}^{u}\left(O_{0}\right)$ which contains the $z$-axis in its closure. Then the set

$$
U\left(z_{1}\right) \cup U\left(z_{2}\right) \cup\left\{(0,0, z) \mid z_{1} \leqslant z \leqslant z_{2}\right\} \cup \phi_{0}\left(\left[z_{1}, z_{2}\right]\right),
$$

is the boundary of $H_{0}$, in manifold sense, see Fig. 3. We have that if $\varepsilon$ is sufficiently small, then $\tilde{\phi}_{0}$ can be homotopically deformed to a curve $\hat{\phi}_{0}$ which lies in $H_{0}$ in such a way that that the deformation leaves the $z$-coordinate unchanged and remains inside the region $\mathcal{S}$.

Indeed, let $\varphi(\mathbf{x}, t)$ denote the solution of (2.3) with $\varphi(\mathbf{x}, 0)=\mathbf{x}$. Let us define the curve $\hat{\phi}_{0}$ in $H_{0}$ as

$$
\hat{\phi}_{0}(z)= \begin{cases}\varphi\left(\tilde{\phi}_{0}(z), \gamma^{-1} \log \frac{z}{z_{1}}\right) & \text { if } z_{1}-\leqslant z<z_{1}+\varepsilon \\ (0,0, z) & \text { if } z_{1}+\varepsilon \leqslant z \leqslant z_{2}-\varepsilon \\ \varphi\left(\tilde{\phi}_{0}(z), \gamma^{-1} \log \frac{z}{z_{2}}\right) & \text { if } z_{2}-\varepsilon<z \leqslant z_{2}\end{cases}
$$

If $\varepsilon$ is small, the distance between $\tilde{\phi}_{0}(z)$ and $\hat{\phi}_{0}(z)$ is uniformly small for $z \in\left[z_{1}, z_{2}\right]$. Fixing such an $\varepsilon$, a homotopy between the two curves with the desired properties is readily constructed. We check next that $\phi_{0}$ and $\hat{\phi}_{0}$ are homotopic inside $H_{0}$, with invariant $z$-coordinate. It is straightforward to check that there is a homeomorphism $F: H_{0} \rightarrow[0,1] \times\left[z_{1}, z_{2}\right]$ which 
leaves the $z$-coordinate invariant and satisfies the following properties

$$
\begin{gathered}
F\left(U_{1}\left(z_{1}\right)\right)=[0,1] \times\left\{z_{1}\right\}, \quad F\left(U_{1}\left(z_{2}\right)\right)=[0,1] \times\left\{z_{2}\right\}, \\
F\left(\left\{(0,0, z) \mid z_{1} \leqslant z \leqslant z_{2}\right\}\right)=\{0\} \times\left[z_{1}, z_{2}\right], \\
F\left(\phi_{0}\left(\left[z_{1}, z_{2}\right]\right)=\{1\} \times\left[z_{1}, z_{2}\right] .\right.
\end{gathered}
$$

On the other hand, it can also be checked that the curves $F\left(\phi_{0}(z)\right)$ and $F\left(\hat{\phi}_{0}(z)\right), z \in\left[z_{1}, z_{2}\right]$ are homotopic inside the rectangle $[0,1] \times\left[z_{1}, z_{2}\right]$, with a homotopy $G$ which leaves the endpoints of these curves as well as their $z$-coordinates invariant. $F^{-1} o G$ is a homotopy in $H_{0}$ with the desired properties. See Fig. 4.

It follows that the linking number of $\phi_{0}$ and $\phi_{\infty}$ equals that of $\tilde{\phi}_{0}$ and $\phi_{\infty}$.

Let us write

$$
\phi_{\infty}\left(z_{1}\right)=\left(0, y_{1}, z_{1}\right), \quad \phi_{\infty}\left(z_{2}\right)=\left(x_{2}, y_{2}, z_{2}\right) .
$$

We define $\tilde{\phi}_{\infty}$ as

$$
\tilde{\phi}_{\infty}(z)=\left\{\begin{array}{l}
\left(0, y_{1}, z\right) \quad \text { if } z_{1} \leqslant z<z_{1}+\varepsilon \\
\left(0, y_{1}, z_{1}+\varepsilon\right)+\frac{z-z_{1}-\varepsilon}{z_{2}-z_{1}-2 \varepsilon}\left(x_{2}, y_{2}-y_{1}, z_{2}-z_{1}-2 \varepsilon\right) \\
\quad \text { if } z_{1}+\varepsilon \leqslant z \leqslant z_{2}-\varepsilon \\
\left(x_{2}, y_{2}, z\right) \quad \text { if } z_{2}-\varepsilon<z \leqslant z_{2} .
\end{array}\right.
$$
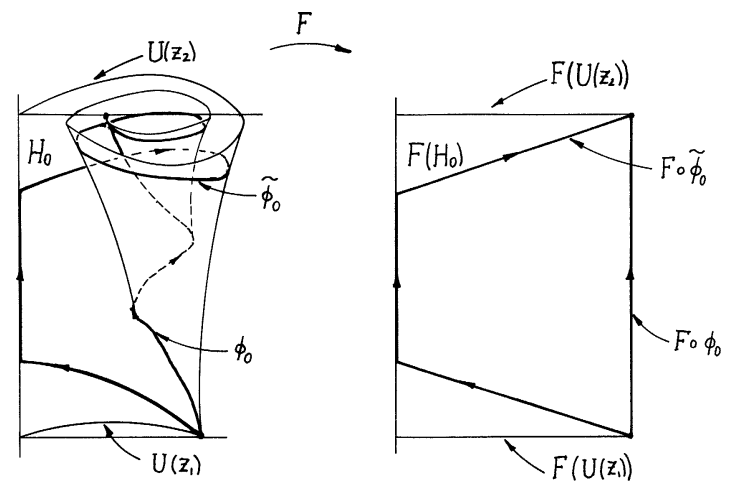

Fig. 4. 
Then $\phi_{\infty}$ and $\tilde{\phi}_{\infty}$ are homotopic inside

$$
\left\{z_{1} \leqslant z \leqslant z_{2}\right\} \backslash \tilde{\phi}_{0}\left(\left[z_{1}, z_{2}\right]\right),
$$

leaving endpoints fixed and $z$-coordinate invariant. Indeed, if we choose $\delta$ and $\varepsilon$ sufficiently small, we obtain that $\phi_{\infty}\left(\left[z_{1}, z_{2}\right]\right)$ and $\tilde{\phi}_{\infty}\left(\left[z_{1}, z_{2}\right]\right)$ are contained in the set

$$
\begin{aligned}
\mathcal{R}= & \left\{\left|(x, y)-\left(0, y_{1}\right)\right|<\delta, z_{1} \leqslant z<z_{1}+\varepsilon\right\} \\
& \cup\left\{x>0, z_{1}+\varepsilon \leqslant z<z_{2}-\varepsilon\right\} \\
& \cup\left\{\left|(x, y)-\left(x_{2}, y_{2}\right)\right|<\delta, z_{2}-\varepsilon<z \leqslant z_{2}\right\},
\end{aligned}
$$

and $\tilde{\phi}_{0}\left(\left[z_{1}, z_{2}\right]\right) \cap \mathcal{R}=\emptyset$. It is easily checked that a homotopy in $\mathcal{R}$ with the desired properties can be built up.

Hence the linking number of the curves $\phi_{0}, \phi_{\infty}$ in $\left[z_{1}, z_{2}\right]$ equals that of $\tilde{\phi}_{0}$ and $\tilde{\phi}_{\infty}$.

We claim that the winding number of $\sigma_{2}$ around the point $\phi_{\infty}\left(z_{2}\right)$, measured in the plane $z=z_{2}$. is greater than or equal to $m-1$ and less than or equal to $m$, where $m$ is the linking number of $\tilde{\phi}_{0}$ and $\tilde{\phi}_{\infty}$. We have that

$$
\begin{aligned}
& \tilde{\phi}_{0}(z)-\tilde{\phi}_{\infty}(z) \\
& \quad= \begin{cases}\sigma_{1}\left(\frac{z-z_{1}}{\varepsilon}\right)-\left(0, y_{1}, z_{1}\right) & \text { if } z_{1} \leqslant z<z_{1}+\varepsilon, \\
-\left(0, y_{1}, 0\right)-\frac{z-z_{1}-\varepsilon}{z_{2}-z_{1}-2 \varepsilon}\left(x_{2}, y_{2}-y_{1}, 0\right) & \text { if } z_{1}+\varepsilon \leqslant z \leqslant z_{2}-\varepsilon, \\
\sigma_{2}\left(\frac{z-\left(z_{2}-\varepsilon\right)}{\varepsilon}\right)-\left(x_{2}, y_{2}, z_{2}\right) & \text { if } z_{2}-\varepsilon<z \leqslant z_{2} .\end{cases}
\end{aligned}
$$

Let us call $\tilde{\phi}(z)$ the $x-y$ component of $\tilde{\phi}_{0}(z)-\tilde{\phi}_{\infty}(z)$. Let us write $\tilde{\phi}(z)=(\rho(z) \sin \theta(z), \rho(z) \cos \theta(z))$. We observe that $W(\tilde{\phi},(0,0))$ corresponds precisely to the linking number of $\tilde{\phi}_{0}$ and $\tilde{\phi}_{\infty}$,

$$
\begin{aligned}
W(\tilde{\phi},(0,0))= & {\left[\frac{1}{2 \pi}\left(\theta\left(z_{2}\right)-\theta\left(z_{1}\right)\right)\right] } \\
= & {\left[\frac { 1 } { 2 \pi } \left(\theta\left(z_{1}+\varepsilon\right)-\theta\left(z_{1}\right)+\theta\left(z_{2}-\varepsilon\right)-\theta\left(z_{1}+\varepsilon\right)\right.\right.} \\
& \left.+\theta\left(z_{2}\right)-\theta\left(z_{2}-\varepsilon\right)\right] .
\end{aligned}
$$

We claim that

$$
\left|\theta\left(z_{1}+\varepsilon\right)-\theta\left(z_{1}\right)\right|<\pi
$$


and

$$
\left|\theta\left(z_{2}-\varepsilon\right)-\theta\left(z_{1}+\varepsilon\right)\right|<\pi .
$$

We check first (3.2). We recall that $\sigma_{1}$ is a parametrization of $U\left(z_{1}\right)=$ $W_{+}^{u}\left(O_{0}\right) \cap\left\{z=z_{1}\right\}$. Because of the form of vector field defining system (2.3), $W_{+}^{u}\left(O_{0}\right)$ cannot intersect the set $\{x=0, y>0\}$. On the other hand, $W^{u}\left(O_{0}\right)$ splits into a half-plane $H$ contained in $\{x<0, y<0\}$ and $W_{+}^{u}\left(O_{0}\right)$. From these facts it follows that $U\left(z_{1}\right)$ does not intersect $\{x<0, y>0\}$ and hence (3.2) holds true. Now, since between $z_{1}+\varepsilon$ and $z_{2}-\varepsilon$, the curve $\phi(z)$ is a line segment, inequality (3.3) readily follows. From (3.2), (3.3) and (3.1) it follows that

$$
n \leqslant W(\tilde{\phi},(0,0)) \leqslant n+1
$$

where

$$
n=\left[\frac{1}{2 \pi}\left(\theta\left(z_{2}\right)-\theta\left(z_{2}-\varepsilon\right)\right)\right] .
$$

But $n$ is precisely the winding number we want to estimate and $W(\tilde{\phi},(0,0))=m$. Thus the claim follows, and hence the lemma.

Proof of Lemma 3.2. - We will show that the linking number of $\phi_{0}$ and $\phi_{\infty}$ in $\left[z_{1}, z_{2}\right]$ where exactly $2 k+1$ zeros of $x_{0}-x_{\infty}$ exist in the interval $\left[t_{-}, t_{+}\right]$and they are in the interior of the interval, is at least $k$. Here $t_{ \pm}=\gamma^{-1} \log z_{1,2}$.

Let $h=x_{0}-x_{\infty}$. Then $h$ satisfies a second order equation of the form

$$
h^{\prime \prime}+\alpha h^{\prime}+a(t) h=0
$$

and $h$ has exactly $2 k+1$ zeros in $\left(t_{-}, t_{+}\right)$. By definition of the linking number, it equals the winding number around the origin of the curve $\sigma(z)=\left(h, h^{\prime}\right)\left(\gamma^{-1} \log z\right)$ in $\left[z_{1}, z_{2}\right]$. But this number is invariant under a reparametrization of the curve $\sigma$, it hence equals that of $\hat{\sigma}(t)=\left(h, h^{\prime}\right)(t)$, $t \in\left[t_{-}, t_{+}\right]$. Note that this curve does not touch the point $(0,0)$ since $h$ cannot have a double zero, hence the winding number $W(\hat{\sigma},(0,0))$ is indeed well defined. Let us also observe that whenever $h$ vanishes, $\sigma$ crosses transversally the line $h=0$ in the clockwise direction. Let $t_{-}<t_{1}<t_{2}<\cdots<t_{2 k+1}<t_{+}$be the zeroes of $h$, and consider a lifting $(\rho(t), \theta(t))$ of $\hat{\sigma}$, so that $\hat{\sigma}(t)=(\rho(t) \sin \theta(t), \rho(t) \cos \theta(t))$. Assume $\theta\left(t_{-}\right) \in(0, \pi)$. Then $\theta\left(t_{j}\right)=j \pi$ for $j=1, \ldots, 2 k+1$. Since no more zeros exist beyond $t_{2 k+1}$, it follows that $(2 k+1) \pi<\theta\left(t_{+}\right)<(2 k+2) \pi$. 
and hence

$$
W(\hat{\sigma},(0,0))=\left[\frac{1}{2 \pi}\left(\theta\left(t_{+}\right)-\theta\left(t_{-}\right)\right)\right]=k,
$$

as desired. The proof if $\theta\left(t_{-}\right) \in(\pi, 2 \pi)$ is similar.

Remark. - We observe from the above proof that the linking number of $\phi_{0}$ and $\phi_{\infty}$ is nondecreasing as a function of the interval where it is measured, namely the linking number in $\left[z_{1}^{\prime}, z_{2}^{\prime}\right]$ is larger than or equal to that in $\left[z_{1}, z_{2}\right]$ whenever $\left[z_{1}, z_{2}\right] \subset\left[z_{1}^{\prime}, z_{2}^{\prime}\right]$.

Proof of Lemma 3.3. - transformation of $\mathbf{x}_{\infty}$ via (2.4). We recall that for $z_{2}>0$, the section $S\left(z_{2}\right)$ is given by $H_{\infty} \cap\left\{z=z_{2}\right\}$, which is a curve with endpoints $\left(0,0, z_{2}\right)$ and $\mathbf{x}_{\infty}\left(\gamma^{-1} \log z_{2}\right)$. We have that the orbit of $\mathbf{x}_{\infty}$ lies in $W^{s}\left(O_{\infty}\right)$, so that in coordinates (2.4), $\tilde{\mathbf{x}}(t) \rightarrow 0$ as $t \rightarrow+\infty$. Hence if $\tilde{x}_{2}$ is sufficiently small, $\tilde{S}\left(\tilde{z}_{2}\right)$ is well approximated by the segment joining its endpoints. Now, the image of this segment via transformation (2.4) is a line segment joining $\left(0,0, z_{2}\right)$ and $\mathbf{x}_{2}\left(\gamma^{-1} \log z_{2}\right)$, where $z_{2}=\tilde{z}_{2}^{-(p-1) /(q-1)}$. Thus, if $\tilde{z}_{2}$ is small enough, we also have that $S\left(z_{2}\right)$ is well approximated by the segment joining its endpoints.

Let $\eta_{2}(s), s \in[0,1]$ be a parametrization of $S\left(z_{2}\right)$ such that $\eta_{2}(0)=$ $\left(0,0, z_{2}\right), \eta_{2}(1)=\phi_{\infty}\left(z_{2}\right) \equiv\left(x_{2}, y_{2}, z_{2}\right)$. Let us call $\eta$ the vector in $\mathbb{R}^{2}$ whose components are the $x, y$ coordinates of $\eta_{2}$. Since $S\left(z_{2}\right)$ does not have self-intersections, we may choose $\eta$ to be one-to-one. $\eta(s)$, $s \in[0,1)$, can be lifted to a curve $\bar{\eta}(s)=\left(\theta_{\eta}(s), \rho_{\eta}(s)\right)$ in the polar coordinates plane, so that

$$
\eta(s)=\left(\rho_{\eta}(s) \sin \theta_{\eta}(s)+x_{2}, \rho_{\eta}(s) \cos \theta_{\eta}(s)+y_{2}\right) .
$$

Now, as we have mentioned, the $z$-axis separates $W^{u}\left(O_{0}\right)$ into two components, one of them a half-plane contained in $x<0, y<0$ and the other the surface we called $W_{+}^{u}\left(O_{0}\right)$. Thus $U\left(z_{2}\right)$ does not intersect this half-plane. We denote by $l$ the parametrization of $(x, y)$-coordinates of the line constituting the intersection of the half plane and $z=z_{2}$, let us say $l(s)=-(s, a s), s>0$, for some $a>0$. We call $\bar{l}=\left(\theta_{l}, \rho_{l}\right)$ its lifting to polar coordinates around $\left(x_{2}, y_{2}\right)$. Since $\eta(0)=\sigma(0)=l\left(0^{+}\right)=(0,0)$, then $\bar{\eta}(0)=\bar{\sigma}(0)=\bar{l}(0)=\left(\theta_{0}, \rho_{0}\right)$.

Let us call $m$ the integer $m=W\left(\sigma,\left(x_{2}, y_{2}\right)\right)$. Then there exist numbers $0=s_{0}<s_{1}<\cdots<s_{m} \leqslant 1$ such that $\theta_{\sigma}\left(s_{j}\right)=2 j \pi+\theta_{0}$. Now, if $z_{2}$ is sufficiently large, we have that $\sigma(1) \in\{x<0\}$ and we can conclude $\theta_{\sigma}(1) \geqslant 2(m+1) \pi+\theta_{0}$. Thus, if we set $s_{m+1}=1$, then $s_{m}<s_{m+1}$. We 
will show that for each $1 \leqslant j \leqslant m \bar{\sigma}\left(\left(s_{j-1}, s_{j+1}\right)\right)$ intersects the curve $\bar{\eta}(s)+(2 j \pi, 0)$.

Let us observe that since $\eta$ is well approximated by the segment joining $(0,0)$ and $\left(x_{2}, y_{2}\right)$, then

$$
\theta_{0}-\pi<\theta_{\eta}(s)<\theta_{0}+\pi \text { for all } s \in[0,1) .
$$

We also have that

$$
\theta_{0}-\pi<\theta_{l}(s)<\theta_{0}+\pi \quad \text { for all } s \in(0, \infty) \text {. }
$$

Besides, $\rho_{l}(s) \rightarrow \infty$ if $s \rightarrow+\infty$. Thus, the curve $L_{j}$ obtained by joining the curves $\bar{\eta}+(2 j \pi, 0)$ and $\bar{l}+(2 j \pi, 0)$ is contained in the set

$$
\left((2 j-1) \pi+\theta_{0},(2 j+1) \pi+\theta_{0}\right) \times(0, \infty) .
$$

$L_{j}$ does not have self-intersections, so that it separates the half-plane $\rho>0$ into two components, one of them containing the set $\{\theta \leqslant(2 j-$ $\left.1) \pi+\theta_{0}\right\}$ and the other $\left\{\theta \geqslant(2 j+1) \pi+\theta_{0}\right\}$. Therefore, for all $1 \leqslant$ $j \leqslant m, \bar{\sigma}\left(s_{j}, s_{j+1}\right)$ intersects $L_{j}$. Since $\sigma$ does not intersect $l, \bar{\sigma}$ does not intersect $\bar{l}+(2 j \pi, 0)$. Hence $\bar{\sigma}\left(s_{j}, s_{j+1}\right)$ intersects $\bar{\eta}+(2 j \pi, 0)$, and the claim is thus proven, see Fig. 5. Next we see that these correspond to distinct intersections $\mathrm{n}$ the original coordinates. Now, let $a_{j} \in\left(s_{j-1}, s_{j+1}\right)$

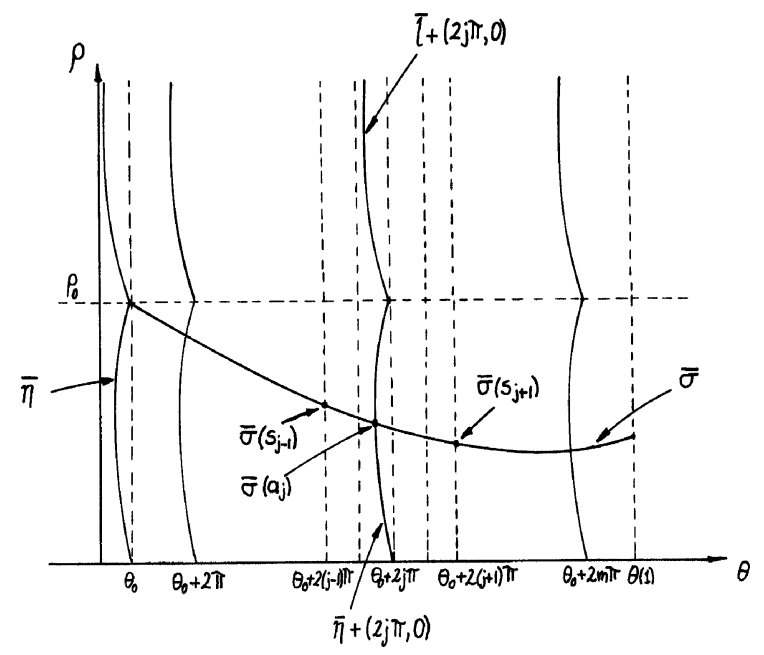

Fig. 5. 
be such that $\bar{\sigma}\left(a_{j}\right)$ lies on the curve $\bar{\eta}+(2 j \pi, 0)$. We have that if $j_{1} \neq j_{2}$ then $\bar{\sigma}\left(a_{j_{1}}\right) \neq \bar{\sigma}\left(a_{j_{2}}\right)$. In fact, if otherwise, the curves $\bar{\eta}+\left(2 j_{1} \pi, 0\right)$ and $\bar{\eta}+\left(2 j_{2} \pi, 0\right)$ would intersect, and then the curve $\eta$ would self intersect, and this does not happen. Thus, for all $j$, there is a $b_{j}$ such tat $\bar{\sigma}\left(a_{j}\right)=\bar{\eta}\left(b_{j}\right)+(2 j \pi, 0)$, so that $\sigma\left(a_{j}\right)=\eta\left(b_{j}\right)$. Since $\sigma$ is one-toone, all points $\sigma\left(a_{j}\right)$ are distinct, and hence $U\left(z_{2}\right)$ and $S\left(z_{2}\right)$ intersect at least at $m-1$ points. This concludes the proof.

\section{THE PROOF OF THEOREM 1.1}

Let us consider first the situation described in Theorem 1.1. We fix a number $q$ with $q>(N+2) /(N-2)$. In view of Proposition 1.1 it is sufficient to establish that, given $k \geqslant 1$, there is a number $p_{k}<$ $(N+2) /(N-2)$ such that for $p_{k}<p<(N+2) /(N-2)$ there exist solutions of (1.4) $u_{1}$ and $u_{2}$ with $u_{1}$ positive in $\left(0, R_{1}\right)$ and $u_{2}$ positive in $\left(R_{2}, \infty\right)$ with the property that $u_{1}-u_{2}$ is not identically zero and has at least $2 k+1$ zeros. To do this, we establish first the following fact.

Lemma 4.1. - Assume $p=(N+2) /(N-2)$ and $q>p$. Let $(x(t)$, $y(t), z(t))$ be any trajectory in $W_{u}\left(O_{0}\right)$ with $z(t)>0$ and $x(t)>0$ as $t \rightarrow-\infty$. Then

(i) $x(t)>0$ for all $t>0$.

(ii) $\tilde{x}(t)$ defined by transformation (2.4) is uniformly bounded and remains away from zero as $t \rightarrow \infty$.

Proof. - Consider such a trajectory and let $\tilde{x}(t)$ be defined by the transformation (2.4), for this $q$ and for $p=(N+2) /(N-2)$. Then $\tilde{x}$ satisfies the second order equation

$$
\tilde{x}^{\prime \prime}+\tilde{x}_{+}^{(N+2) /(N-2)}+\mathrm{e}^{-\tilde{\gamma} t} \tilde{x}_{+}^{q}-\tilde{\beta} \tilde{x}=0, \quad-\infty<t<+\infty .
$$

Assume that $\tilde{x}$ vanishes at a first point $t=T$. Observe that $\tilde{x}(t)=$ $C \mathrm{e}+\mathrm{o}(\mathrm{e})$ as $t \rightarrow \infty$ for some $C>0$. Integrating Eq. (4.1) between $-\infty$ and $T$, after multiplying by $\tilde{x}^{\prime}$ we obtain

$$
\frac{\tilde{x}^{\prime}(T)^{2}}{2}+\int_{-\infty}^{T} \mathrm{e}^{-\tilde{\gamma} t} \tilde{x}_{+}(t)^{q} \tilde{x}^{\prime}(t) \mathrm{d} t=0,
$$


so that after integration by parts we obtain

$$
\frac{\tilde{x}^{\prime}(T)^{2}}{2}+\frac{\tilde{\gamma}}{q+1} \int_{-\infty}^{T} \mathrm{e}^{-\tilde{\gamma} t} \tilde{x}_{+}(t)^{q+1} \mathrm{~d} t=0
$$

and this is a contradiction, unless $x \equiv 0$. This proves the first assertion. For the second assertion, let us assume that there is a sequence $t_{n} \rightarrow+\infty$ so that $\tilde{x}\left(t_{n}\right) \rightarrow 0$. Now one gets the identity

$$
\begin{aligned}
& \frac{\tilde{x}\left(t_{n}\right)^{p+1}}{p+1}+\frac{\tilde{x}^{\prime}\left(t_{n}\right)^{2}}{2}+\mathrm{e}^{-\tilde{\gamma} t_{n}} \frac{\tilde{x}_{+}\left(t_{n}\right)^{q+1}}{q+1}-\tilde{\beta} \frac{\tilde{x_{+}}\left(t_{n}\right)^{2}}{2} \\
& +\frac{\tilde{\gamma}}{q+1} \int_{-\infty}^{t_{n}} \mathrm{e}^{-\tilde{\gamma} t} \tilde{x}_{+}(t)^{q+1} \mathrm{~d} t=0 .
\end{aligned}
$$

Letting $n \rightarrow \infty$ we obtain that

$$
\int_{-\infty}^{\infty} \mathrm{e}^{-\tilde{\gamma} t} \tilde{x}_{+}(t)^{q+1} \mathrm{~d} t=0
$$

hence $\tilde{x} \equiv 0$, a contradiction. Observe that a similar identity also shows that $\tilde{x}$ is also uniformly bounded.

Let $\mathbf{x}_{*}(t)$ be the only trajectory of (2.3) with $z$-component $\mathrm{e}^{\gamma t}$ whose orbit coincides with $W^{s}\left(P_{\infty}\right)$.

Consider also any (fixed) trajectory $\mathbf{x}(t)$ in $W^{u}\left(O_{0}\right)$ which does not coincide with $\mathbf{x}_{*}(t)$. Let $\tilde{x}_{*}(t)$ and $\tilde{x}(t)$ be their respective first coordinates in the transformation (2.4). We claim that $\tilde{x}-\tilde{x}_{*}$ has an infinite number of zeros. In fact, let $t_{n}$ be any sequence with $t_{n} \rightarrow+\infty$. Let us set $\tilde{x}_{n}(t)=\tilde{x}\left(t_{n}+t\right)$. Then from the previous lemma, $\tilde{x}_{n}(t)$ is uniformly bounded above, and below away from zero. $\tilde{x}_{n}$ satisfies the equation

$$
\tilde{x}_{n}^{\prime \prime}+\tilde{x}_{n}^{(N+2) /(N-2)}+\mathrm{e}^{-\tilde{\gamma} t} \delta_{n} \tilde{x}_{n}^{q}-\tilde{\beta} \tilde{x}_{n}=0, \quad-\infty<t<+\infty,
$$

with $\delta_{n}=\mathrm{e}^{-\gamma t_{n}} \rightarrow 0$. By a standard compactness argument, it follows that, passing to a subsequence $\tilde{x}_{n} \rightarrow \bar{x}$, uniformly on compact intervals, where $\bar{x}$ solves

$$
\bar{x}^{\prime \prime}+\bar{x}^{(N+2) /(N-2)}-\tilde{\beta} \bar{x}=0, \quad-\infty<t<+\infty,
$$


$\bar{x}$ is bounded above and below away from zero. Besides, since $\tilde{x}$ and $\tilde{x}_{*}$ do not coincide, and $\tilde{x}_{*}$ is the only trajectory in $W_{u}\left(P_{\infty}\right)$ and $\tilde{x}_{*}\left(t+t_{n}\right) \rightarrow$ $\beta^{1 /(p-1)}$ uniformly on compacts, then $\bar{x}$ is nonconstant. But the only solutions positive and bounded away from zero of the above equation are periodic, and cross the constant value $\beta^{1 /(p-1)}$ an infinite number of times. This proves the claim.

Let us consider an interval $\left[t_{1}, t_{2}\right]$ where one sees $2 k+1$ zeros of $\tilde{x}-\tilde{x}_{1}$. For fixed $q$, we take a number $p$ slightly smaller than $(N+2) /(N-2)$. Then in the coordinates, $W^{s}\left(P_{\infty}\right)$ remains as close as we wish on each given compact interval of the $\tilde{z}$-coordinate to the trajectory $\overline{\tilde{x}}_{1}$ if one chooses $p$ close enough to critical. Similarly, one can find a trajectory in $W^{u}\left(O_{0}\right)$ very close to $\tilde{\mathbf{x}}$ for all $p$ near critical. Since the $2 k+1$ zeros of $\tilde{x}-\tilde{x}_{1}$ are simple, the same will be true for those close-by trajectories, in the interval $\left(t_{1}, t_{2}\right)$ for $p$ sufficiently close to critical. In this way, the assumption of Proposition 1.1 do hold in the situation described in (a) of Theorem 1.1 and the result hence follows. The proof of assertion (b) is actually symmetric. It can be understood as basically a reflection of the situation just described. We need the following analogue of Lemma 4.1.

LEMMA 4.2. - Assume $q=(N+2) /(N-2)$ and $N /(N-2)<p<$ $q$. Let $(x(t), y(t), z(t))$ be any trajectory in $W^{s}\left(O_{\infty}\right)$ with $z(t)>0$ and $x(t)>0$ as $t \rightarrow+\infty$. Then

(i) $x(t)>0$ for all $t>0$.

(ii) $x(t)$ is uniformly bounded and remains away from zero as $t \rightarrow$ $-\infty$.

Proof. $-x(t)$ satisfies the second order differential equation

$$
x^{\prime \prime}+x_{+}^{(N+2) /(N-2)}+\mathrm{e}^{\gamma t} x_{+}^{p}-\beta x=0, \quad-\infty<t<+\infty .
$$

Notice that $\beta>0$ since $p>N /(N-2)$. Assume that $x$ vanishes at a last point $t=T$. Observe that $\tilde{x}(t)=C \mathrm{e}^{d t}+\mathrm{o}\left(\mathrm{e}^{d t}\right)$ as $t \rightarrow-\infty$ for certain numbers $C>0$ and $d>0$. Integrating Eq. (4.4) between $T$ and $\infty$, after multiplying by $x^{\prime}$, and then integrating by parts we now obtain

$$
\frac{x^{\prime}(T)^{2}}{2}+\frac{\gamma}{p+1} \int_{T}^{\infty} \mathrm{e}^{\gamma t} x_{+}^{p+1}(t) \mathrm{d} t=0,
$$

and this is a contradiction, unless $x \equiv 0$. This proves (a). The proof of (b) is analogous to the corresponding assertion in Lemma 4.1. 
After this result, the proof of part (b) of the theorem follows by a similar perturbation analysis as that carried out in part (a), except that now we consider $t \rightarrow-\infty$. This concludes the proof of Theorem 1.1.

\section{THE PROOF OF THEOREM 1.2}

In this section we will perform the proof of the nonexistence result Theorem 1.2. Thus, we fix $q>(N+2) /(N-2)$ and show that if $p$ is taken sufficiently close to $N /(N-2)$, then no ground states of (1.1) (singular or nonsingular) exist.

We consider the initial value problem

$$
\begin{gathered}
u^{\prime \prime}+\frac{N-1}{r} u^{\prime}+u_{+}^{p}+u_{+}^{q}=0, \quad r>0 \\
u^{\prime}(0)=0, \quad u(0)=\alpha>0 .
\end{gathered}
$$

Let $u_{\alpha}(r)$ be the unique solution of this initial value problem. Let us denote by $x_{\alpha}(t)$ and $\tilde{x}_{\alpha}(t)$ their Emden-Fowler transformations, namely

$$
x_{\alpha}(t)=\mathrm{e}^{2 t /(q-1)} u_{\alpha}\left(\mathrm{e}^{t}\right), \quad \tilde{x}_{\alpha}(t)=\mathrm{e}^{2 t /(p-1)} u_{\alpha}\left(\mathrm{e}^{t}\right) .
$$

Then $x_{\alpha}(t)$ has associated a trajectory of system (2.3) in $W^{u}\left(O_{0}\right), \mathbf{x}_{\alpha}(t)=$ $\left(x_{\alpha}(t), y_{\alpha}(t), \mathrm{e}^{\gamma t}\right)$. Let us also consider the unique trajectory $\mathbf{x}_{*}(t)$ with $z$ component $\mathrm{e}^{\gamma t}$, corresponding to the one-dimensional unstable manifold of $P_{0}, W^{u}\left(P_{0}\right)$. Associated to this is then the (unique) singular solution of (5.1) given by $u_{\infty}(r)=r^{-2 /(q-1)} x_{*}(\log r)$, where $x_{*}$ is the $x$-component of $\mathbf{x}_{*}$.

We need the following result.

LEMMA 5.1.-

$$
\lim _{\alpha \rightarrow+\infty} x_{\alpha}(t)=x_{*}(t)
$$

uniformly on compact intervals.

Proof. - We know that the tangent plane to the manifold $W^{u}\left(O_{0}\right)$ is the plane which contains the $z$-axis and the vector $(1,2 /(q-1), 0)$. By Hartman and Grobman Theorem, see Theorem 1.1.3 in [3], we know that the dynamics near $O_{0}$ of the system is characterized by $C^{0}$-conjugation with that of the linear system

$$
x^{\prime}=y, \quad y^{\prime}=\alpha y-\beta x, \quad z^{\prime}=\gamma z,
$$


whose unstable manifold of the origin is precisely the above mentioned plane. Thus there is a unique orbit of the linear system, let us say

$$
\hat{\mathbf{x}}(t)=\hat{\alpha} \mathrm{e}^{2 t /(q-1)}\left(1, \frac{2}{q-1}, 0\right)+\mathrm{e}^{\gamma t}(0,0,1),
$$

whose associated trajectory approximates that of $\mathbf{x}_{\alpha}$. But the initial conditions (5.2) read in terms of this trajectory as

$$
\left(x_{\alpha}(t), y_{\alpha}(t)\right)=\alpha \mathrm{e}^{2 t /(q-1)}\left(1, \frac{2}{q-1}\right)+\mathrm{o}\left(\mathrm{e}^{2 t /(q-1)}\right), \quad \text { as } t \rightarrow-\infty .
$$

Hence $\hat{\alpha}=\alpha$, and the two trajectories remain uniformly close in a neighborhood of the origin independent of $\alpha$. But as $\alpha \rightarrow+\infty$, this trajectory gets closer and closer to the (invariant) plane $z=0$, hence to the heteroclinic orbit contained in $z=0$ which connects $O_{0}$ and $P_{0}$. In particular there are points of the trajectory of $\mathbf{x}_{\alpha}$ which get closer and closer to $P_{0}$ as $\alpha \rightarrow+\infty$. Therefore, for large $\alpha$, this trajectory enters a neighborhood of $P_{0}$ where the dynamics of the system is well described by its linear part. Let us recall that $P_{0}$ is a hyperbolic attractor on the $z=0$ plane, either a focus or a node, while it has one expanding direction transversal to this plane, precisely the tangent line to the onedimensional unstable manifold of $P_{0}$. Examination of the linear system yields that an orbit not contained in the $z=0$ plane which gets close to $P_{0}$, turns upwards, staying close to $W^{u}\left(P_{0}\right)$ in an entire neighborhood of $P_{0}$. Since this neighborhood is independent of $\alpha$, the conclusion is that in a neighborhood of $P_{0}$, the trajectory of $\mathbf{x}_{\alpha}$ gets uniformly close to $W^{u}\left(P_{0}\right)$ as $\alpha \rightarrow+\infty$. Continuity in the initial conditions of the initial value problem associated to the system implies then that given any compact subset of the real line, large alpha implies $\mathbf{x}_{\alpha}$ stays uniformly close to $\mathbf{x}_{*}$. This concludes the proof.

Remark. - The first part of Lemma 2.2 follows immediately from this result. A proof symmetric to the one just carried out, but applied to Eq. (2.5), shows that also $W^{s}\left(P_{\infty}\right)$ is contained in the closure of $W^{s}\left(O_{\infty}\right)$.

LEMmA 5.2. - Given $N /(N-2) \leqslant \bar{p}<(N+2) /(N-2)$ there is a number a such that for all $\alpha \leqslant$ a there is a unique point $t_{\alpha}$ with $\tilde{x}\left(t_{\alpha}\right)=0$. 
Proof. - We consider now directly problem (5.1)-(5.2). Let us set $\tilde{u}_{\alpha}(r)=\alpha^{2 /(p-1)} u_{\alpha}(\alpha r)$. Then $\tilde{u}_{\alpha}$ satisfies

$$
\begin{gathered}
\tilde{u}_{\alpha}^{\prime \prime}+\frac{N-1}{r} \tilde{u}_{\alpha}^{\prime}+\tilde{u}_{\alpha+}^{p}+\alpha^{\tilde{\gamma}} \tilde{u}_{\alpha+}^{q}=0, \quad r>0 \\
\tilde{u}_{\alpha}^{\prime}(0)=0, \quad \tilde{u}_{\alpha}(0)=1 .
\end{gathered}
$$

Since $\alpha^{\tilde{\gamma}} \rightarrow 0$ as $\alpha \rightarrow 0$, it follows by continuity of the solution of this problem in $\alpha$, that $u_{\alpha} \rightarrow u_{0}$ uniformly over compacts, where $u^{*}$ is the unique solution of the initial value problem

$$
\begin{gathered}
u^{\prime \prime}+\frac{N-1}{r} u^{\prime}+u_{+}^{p}=0, \quad r>0 \\
u^{\prime}(0)=0, \quad u(0)=1 .
\end{gathered}
$$

This solution vanishes exactly once at certain number $r^{*}>0$, with $u^{* \prime}\left(r^{*}\right)<0$ since $p$ is subcritical. $r^{*}$ is bounded by some number depending only on $\bar{p}$. Hence for all $\alpha$ sufficiently small, the same will happen at certain point $r_{\alpha}$.

Proof of Theorem 1.2. - Let us fix $q$ supercritical, and consider first the case $p=N /(N-2)$. We claim that no solution of (5.1) positive in the interval $(0, \infty)$ exists in this situation. In this case $\tilde{\beta}=0$, hence the equation satisfied in the ${ }^{\sim}$ coordinates is

$$
\tilde{x}^{\prime \prime}-\tilde{\alpha} \tilde{x}^{\prime}+\tilde{x}^{p} \mathrm{e}^{-\tilde{\gamma} t} \tilde{x}^{q}=0 .
$$

Let us observe that this solution satisfies that $\tilde{x}(t) \rightarrow 0$ and $\tilde{x}^{\prime}(t) \rightarrow 0$ as $t \rightarrow-\infty$, hence integrating the equation from $-\infty$ to $t$ we obtain the relation

$$
\tilde{x}^{\prime}(t)-\tilde{\alpha} \tilde{x}(t)+\int_{-\infty}^{t} \tilde{x}^{p}(\tau) \mathrm{d} \tau \leqslant 0 .
$$

We have that $\tilde{x}(t)$ and $\tilde{x}^{\prime}(t)$ are uniformly bounded. In fact, for instance boundedness of $\tilde{x}$ is equivalent to that of the function $r^{2 /(p-1)} u(r)$. Integrating (5.1) we obtain that

$$
-u^{\prime}(r) \geqslant \frac{1}{r^{N-1}} \int_{0}^{r} u^{p}(s) s^{N-1} \mathrm{~d} s .
$$


In particular $u$ is decreasing, so that,

$$
-u^{\prime}(r) \geqslant \frac{r}{N} u^{p}(r) .
$$

From here it easily follows that $u(r) \leqslant C r^{-2 /(p-1)}$ and $u^{\prime}(r) \leqslant$ $C r^{-(p+1) /(p-1)}$ which imply that $\tilde{x}$ and $\tilde{x}^{\prime}$ are bounded.

Coming back to relation (5.3), we obtain from the boundedness of $\tilde{x}$ and $\tilde{x}^{\prime}$ that $\int_{-\infty}^{\infty} \tilde{x}^{p}(\tau) \mathrm{d} \tau<+\infty$. Hence there is a sequence $t_{n}$ such that $\tilde{x}\left(t_{n}\right) \rightarrow 0$ and $\tilde{x}^{\prime}\left(t_{n}\right) \rightarrow 0$. But, invoking again relation (5.3) at $t=t_{n}$ and letting $n \rightarrow \infty$ we obtain $\int_{-\infty}^{\infty} \tilde{x}^{p}(\tau) \mathrm{d} \tau=0$, hence $\tilde{x} \equiv 0$, a contradiction which proves the claim.

Let us now proceed to the proof of the theorem. From Lemma 5.3, we see that the singular solution $\mathbf{x}_{*}$ crosses transversally the plane $x=0$ at some height $z=\bar{z}$. From Lemma 5.1, it follows that for each $p$ close to $N /(N-2)$ and all $\mathbf{x}_{\alpha}$ 's with sufficiently large $\alpha$, let us say $\alpha \geqslant b>0$, also cross $x=0$ before reaching height $2 \bar{z}$. On the other hand, from Lemma 5.2, we see that all $\tilde{\mathbf{x}}_{\alpha}^{\prime} s$ with sufficiently small $\alpha$, say $0<\alpha<a$, also cross the plane $\tilde{x}=0$ and the distance from the crossing point to the $\tilde{x}$-axis is bounded below, away from zero.

Let us now consider $x_{\alpha}$ with $\alpha \in[a, b]$. From Lemma 5.3, all $x_{\alpha}$ 's vanish before infinity if $p=N /(N-2)$. Continuity of the solution of the initial value problem in $p$ then implies that for all $p$ sufficiently close to $N /(N-2)$, and all $\alpha \in[a, b], x_{\alpha}$ also vanishes. Summarizing, we have shown that no solution of problem (5.1)-(5.2) can remain positive for all $r>0$ if $p$ is sufficiently close to $N /(N-2)$. This concludes the proof of the theorem.

\section{THE PROOF OF THEOREM 1.3}

Let us fix $q>(N+2) /(N-2)$. A first observation is that a singular ground state with slow decay exists if and only if the one dimensional manifolds $W^{u}\left(P_{0}\right)$ and $W^{s}\left(P_{\infty}\right)$ coincide, while a singular ground state with fast decay is present whenever $W^{u}\left(P_{0}\right)$ is contained in $W^{s}\left(O_{\infty}\right)$.

Let us consider the solutions $\mathbf{x}_{0}(t)$ and $\mathbf{x}_{\infty}(t)$ with $z$-component $\mathrm{e}^{\gamma t}$ whose trajectories coincide respectively with $W^{u}\left(P_{0}\right)$ and $W^{s}\left(P_{\infty}\right)$.

Referring to the notations introduced in the proof of Lemma 3.3, we consider for a number $z_{+}>0$ to be fixed later, the unstable and stable sections $U^{p}\left(z_{+}\right)$and $S^{P}\left(z_{+}\right)$. We consider one-to-one parametrizations $\sigma^{p}$ and $\eta^{p}$ with $\sigma^{p}(0)=\eta^{p}(0)=\left(0,0, z_{+}\right)$and $\sigma^{p}(1)=\phi_{0}\left(z_{+}\right) \equiv P^{p}$ 
and $\eta^{p}(1)=\phi_{\infty}\left(z_{+}\right) \equiv Q^{p}$. Let also $l(s)$ be the half line constituted by the $z_{+}$-section of the plane branch of $W^{u}\left(O_{0}\right)$, contained in $x<0$.

Let us consider liftings to polar coordinates around the point $Q^{p}$,

$$
\begin{gathered}
\bar{\sigma}^{p}(s)=\left(\theta_{\sigma}^{p}(s)\right), \rho_{\sigma}^{p}(s), \quad \bar{\eta}^{p}(s)=\left(\theta_{\eta}^{p}(s), \rho_{\eta}^{p}(s)\right), \\
\bar{l}^{p}(s)=\left(\theta_{l}^{p}(s), \rho_{l}^{p}(s)\right)
\end{gathered}
$$

of these curves, selected so that

$$
\left(\theta_{0}(p), \rho_{0}(p)\right) \equiv \bar{\sigma}^{p}(0)=\bar{\eta}^{p}(0)=\bar{l}^{p}(0)
$$

defines a continuous function of $p$.

Let us consider a number $p_{0}>N /(N-2)$ such that

$$
W^{u}\left(P_{0}\right) \cap\left(W^{s}\left(P_{\infty}\right) \cup W^{s}\left(0_{\infty}\right)\right)=\emptyset .
$$

Let $N\left(p_{0}\right)$ be the total linking number in $(0, \infty)$ of the curves $\phi_{0}$ and $\phi_{\infty}$. Then $N\left(p_{0}\right)<+\infty$. From the proof of Theorem 1.1 we know that $N(p)$ grows to infinity as $p \uparrow(N+2) /(N-2)$. Let us choose a number $p_{0}<p_{1}<(N+2) /(N-2)$ with $N\left(p_{1}\right) \geqslant N\left(p_{0}\right)+4$ and such that (6.1) also holds at $p_{1}$. The claim, from which the result of part (a) of the theorem readily follows, is that there must exist a number $p \in\left(p_{0}, p_{1}\right)$ such that either $P^{p}=Q^{p}$ or $P^{p} \in S^{p}\left(z_{+}\right)$. We will show this, making a suitable choice of $z_{+}$.

Let us observe first that there is a number $M>0$ such that for all $p \in\left[p_{0}, p_{1}\right], z_{+} \geqslant 1, s \in[0,1],\left|\rho_{\sigma}^{p}(s)\right| \leqslant M$. On the other hand, since $\mathbf{x}_{0}(t)$ does not correspond to a singular ground state for any $p \in\left[p_{0}, p_{1}\right]$, it must cross the $x=0$ plane. It follows that if we fix $z_{+}$large enough we may also assume that $\left|Q^{p}-P^{p}\right|=\rho_{\sigma}^{p}(1)>M$ for all $p \in\left[p_{0}, p_{1}\right]$. Let us fix such a $z_{+}$.

Let $n_{0}$ be the winding number $n_{0} \equiv W\left(\sigma^{p_{0}}, Q^{p_{0}}\right)$. Then, enlarging $z_{+}$ if necessary, we may also assume from Lemma 3.1 that $N\left(p_{0}\right) \leqslant n_{0} \leqslant$ $N\left(p_{0}\right)+1$. Now, from our choice of $p_{1}$ we then have that

$$
W\left(\sigma^{p_{1}}, Q^{p_{1}}\right) \geqslant n_{0}+3 \text {. }
$$

Let us consider, the translates of the curve $\bar{l}^{p}, \bar{l}_{n}^{p}(s)=\bar{l}^{p}(s)+(2 n \pi, 0)$. Then if $M>0$ was chosen large enough, the curves $l_{n}^{p}$ separate the region $\rho>M$ into connected components, for all $p \in\left[p_{0}, p_{1}\right] . l^{n}(s)$. Now, $\theta_{l}(s) \in\left(\theta_{0}(p)-\pi, \theta_{0}(p)+\pi\right)$. Let us assume that the point $\bar{\sigma}^{p_{0}}(1)$ 


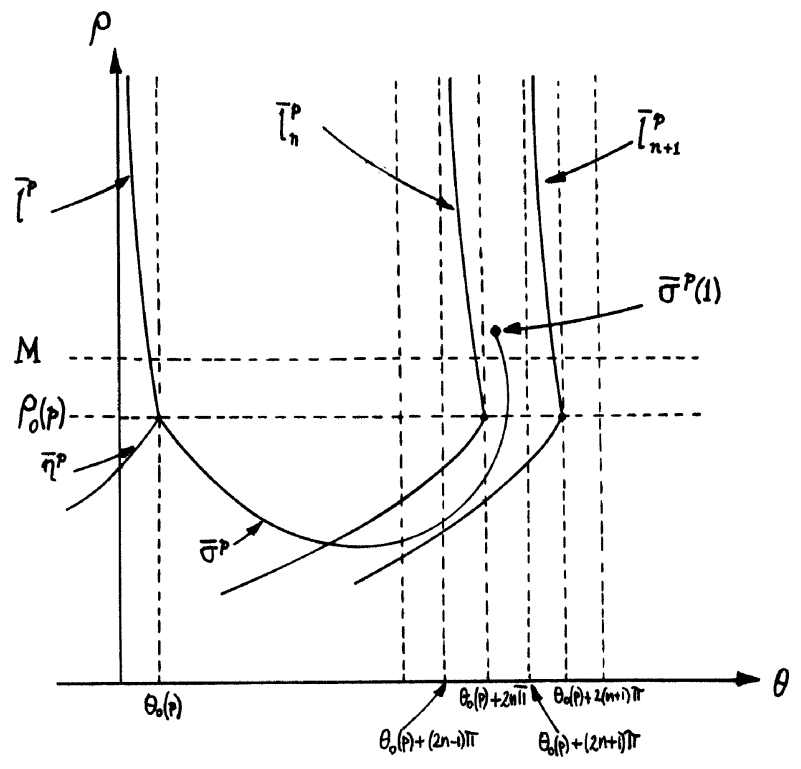

Fig. 6.

was between $l_{n}^{p_{0}}$ and $l_{n+1}^{p_{0}}$. (Actually $n=n_{0}$ or $n=n_{0}-1$.) Then, by continuity, $\bar{\sigma}^{p}(1)$ is between $\bar{l}_{n}^{p}$ and $\bar{l}_{n+1}^{p}$ for all $p \in\left[p_{0}, p_{1}\right]$ since this point always was in $\rho>M$, see Fig. 6 . We conclude that

$$
\theta_{\sigma}^{p_{1}}(1) \leqslant \theta_{0}\left(p_{1}\right)+\pi+2 \pi(n+1) \leqslant 2 \pi\left(n_{0}+2\right),
$$

and hence the winding number

$$
W\left(\sigma^{p_{1}}, Q^{p_{1}}\right) \leqslant n_{0}+2 .
$$

We have reached a contradiction with (6.2), and hence the assertion of the theorem in its part (a) holds. The proof of part (b) of the theorem is analogous.

\section{ACKNOWLEDGEMENTS}

We would like to thank Raúl Manásevich for several useful conversations on the problem treated in this work.

R. Bamón has been supported by FONDECYT grant 196-0848, I. Flores by a CONICYT doctoral fellowship, and M. del Pino by FONDECYT grant 100-0969 and FONDAP-Conicyt. 


\section{REFERENCES}

[1] Clemons C.B., Jones C.K.R.T., A geometric proof of the Kwong-McLeod uniqueness result, SIAM J. Math. Anal. 24 (1993) 436-443.

[2] Fowler R., Further studies of Emden's and similar differential equations, Quart. J. Math. 2 (1931) 259-288.

[3] Guckenheimer J., Holmes P., Nonlinear Oscillations, Dynamical Systems and Bifurcations of Vector Fields, Springer-Verlag, New York, 1983.

[4] Gidas B., Ni W.-M., Nirenberg L., Symmetry properties of positive solutions of nonlinear elliptic equations in $\mathbb{R}^{N}$, Adv. Math. Studies 7A (1981) 369-402.

[5] Gidas B., Spruck J., Global and local behavior of positive solutions of nonlinear elliptic equations, Comm. Pure Appl. Math. 34 (1981) 525-598.

[6] Gidas B., Caffarelli L., Spruck J., Asymptotic symmetry and local behavior of semilinear elliptic equations with critical Sobolev growth, Comm. Pure Appl. Math. 42 (3) (1989) 271-297.

[7] Hirsch M., Pugh C., Schub M., Invariant Manifolds, Lecture Notes in Math., Vol. 583, Springer-Verlag, New York, 1977.

[8] Johnson R., Pan X., Yi Y., The Melnikov method and elliptic equations with critical exponent, Indiana Univ. Math. J. 43 (1994) 1045-1077.

[9] Johnson R., Pan X., Yi Y., Positive solutions of super-critical elliptic equations and asymptotics, Comm. Partial Differential Eqnuations 18 (1993) 977-1019.

[10] Lin C.-S., Ni W.-M., A counterexample to the nodal line conjecture and a related semilinear equation, Proc. Amer. Math. 102 (2) (1988) 271-277.

[11] Smoller J., Shock Waves and Reaction Diffusion Equations, 2nd edn., SpringerVerlag, New York, 1994. 